\title{
On the Entropy of Deformed Phase Space Black Hole and the Cosmological Constant
}

\author{
Andrés Crespo-Hernández ${ }^{1,+}$, Eri A. Mena-Barboza ${ }^{1, *,+}$ and Miguel Sabido ${ }^{2,+}$ \\ 1 Centro Universitario de la Ciénega, Universidad de Guadalajara, Edificio P, Ocotlán, Jalisco 47820, Mexico; \\ emena@cuci.udg.mx \\ 2 Departamento de Física de la Universidad de Guanajuato, León 37150, Mexico; msabido@fisica.ugto.mx \\ * Correspondence: emena@cuci.udg.mx; Tel.: +52-392-925-9400 \\ + These authors contributed equally to this work.
}

Academic Editor: Remo Garattini

Received: 27 November 2016; Accepted: 22 February 2017; Published: 28 February 2017

\begin{abstract}
In this paper we study the effects of noncommutative phase space deformations on the Schwarzschild black hole. This idea has been previously studied in Friedmann-Robertson-Walker (FRW) cosmology, where this "noncommutativity" provides a simple mechanism that can explain the origin of the cosmological constant. In this paper, we obtain the same relationship between the cosmological constant and the deformation parameter that appears in deformed phase space cosmology, but in the context of the deformed phase space black holes. This was achieved by comparing the entropy of the deformed Schwarzschild black hole with the entropy of the Schwarzschild-de Sitter black hole.
\end{abstract}

Keywords: black hole entropy; deformed phase space; cosmological constant

\section{Introduction}

The cosmological constant problem has been addressed for several years and remains as one of the central issues in physics [1]. The discovery of the acceleration of the universe is usually attributed to a small non-vanishing cosmological constant $\Lambda$. The different contributions to the vacuum energy density from ordinary particle physics gives a value for $\langle\rho\rangle$ of order $M_{p}^{4}$, and should be canceled by the bare value of $\Lambda$. This cancellation has to be better than $10^{-120}$ if we compare the zero-point energy of a scalar field with the experimental value of $\left\langle\rho_{o b s}\right\rangle$. This incredible degree of fine tuning suggests that we are missing important physics [2]. It is likely that the correct way to interpret the tiny value of the cosmological constant by conventional quantum field theory is not the whole story. This has led many authors to suggest that the solution will come from an unconventional approach in fundamental physics. Recently, there has been mounting evidence of a late time accelerating universe, when one considers deformed phase space models [3-6]. One is tempted to seriously consider the idea of noncommutative space-time to study the cosmological constant [7].

The idea of a noncommutative space-time is not new; it has been studied from the physics perspective by Snyder [8,9] and the formal mathematical perspective was given by Connes [10]. During the last decades, much work has been done in noncommutative physics [11-20]. The noncommutative relationship between the space-time coordinates $\left[\hat{x}^{i}, \hat{x}^{j}\right]=i \Theta^{i j}$ gives an uncertainty principle between the coordinates that can be interpreted as length limit. Furthermore, noncommutativity is expected to be relevant at the Planck scale, where it is known that usual semi-classical considerations break down.

From these ideas, noncommutative theories of gravity were proposed [14-19]. All of these formulations showed that the end result of a noncommutative theory of gravity is a highly nonlinear theory. To avoid the difficulties of working with these highly nonlinear theories, and to study the effects of noncommutativity on different aspects of the universe, noncommutative cosmology 
was proposed [21]. The authors noticed that the noncommutative deformations modify the noncommutative fields, and conjectured that the effects of the full noncommutative theory of gravity should be reflected in the minisuperspace variables. This was achieved by introducing the Moyal product of functions in the Wheeler-DeWitt (WDW) equation of the Kantowski-Sachs (KS) cosmological model, in the same manner as is done in noncommutative quantum mechanics. This approach to noncommutativity has been used in connection to the Hierarchy problem and to the cosmological constant problem $[7,22]$. In the last five years, more general minisuperspace deformations have been used in cosmology. In several models, the authors find an explicit relationship between the cosmological constant $\Lambda$ and the deformation parameter $\eta$, and conjecture that the deformation parameter plays the role of a cosmological constant [4-6]. They arrive at this conclusion by comparing the scale factor of the deformed ("noncommutative") model with the scale factor of a commutative model that includes $\Lambda$, and show that the deformation parameter plays the role of the cosmological constant of the commutative model.

To further explore this relationship, we consider a different gravitational system-the black hole. Black holes are the perfect systems to study fundamental problems in gravity, and therefore natural candidates to further explore the relationship between the cosmological constant and noncommutativity. In our approach, we intend to study the origin of the cosmological constant and show that minisuperspace deformations are a feasible mechanism that can be responsible for its origin.

The main goal of this paper is to see if a relationship between the deformation parameter $\eta$ and the cosmological constant $\Lambda$ arises in the context of deformed phase space black holes. We will introduce noncommutativity in the phase space constructed from the minisuperspace variables by modifying the symplectic structure (Poisson's algebra of the minisuperspace) [4-6,21,22]. To calculate the entropy of the deformed black hole, we follow the procedure in $[23,24]$, where the authors study noncommutativity in black holes. We start with the phase space deformation [4-6,22] in the KS cosmological model [23,25]. Interchanging the coordinates $t \leftrightarrow r$, we go from a cosmological solution to a black hole solution $[23,24]$ and find the WDW equation for the noncommutative Schwarzschild black hole, as we are interested in obtaining the modification to the entropy of the black hole due to the phase space deformation. We use the Feynman-Hibbs path integrals formalism [26] to calculate the thermodynamical properties of the black hole [23,27]. In the spirit of [4-6], we compare the entropy of the deformed model with the entropy of a commutative model (Schwarzschild-de Sitter black hole) to find a relationship between $\Lambda$ and the deformation parameters.

The paper is organized as follows: in Section 2, we present the model and introduce the noncommutative deformation. In Section 3, we will show that in our approach, the origin of the cosmological constant and the deformation parameters are related. Section 4 is devoted to final remarks.

\section{Deformed Black Hole Model}

In this section, we present the model for the deformed phase space black hole. It is based on the relationship between the cosmological KS metric and the Schwarzschild metric [28]. The Schwarzschild solution can be written as

$$
d s^{2}=-\left(1-\frac{2 m}{r}\right) d t^{2}+\left(1-\frac{2 m}{r}\right)^{-1} d r^{2}+r^{2}\left(d \theta^{2}+\sin ^{2} \theta d \varphi^{2}\right)
$$

For the case $r<2 m$, the $g_{t t}$ and $g_{r r}$ components of the metric change their sign and $\partial_{t}$ becomes a space-like vector. If we make the coordinate transformation $t \leftrightarrow r$, we find

$$
d s^{2}=-\left(\frac{2 m}{t}-1\right)^{-1} d t^{2}+\left(\frac{2 m}{t}-1\right) d r^{2}+t^{2}\left(d \theta^{2}+\sin ^{2} \theta d \varphi^{2}\right)
$$


when compared with the parametrization by Misner of the KS metric

$$
d s^{2}=-N^{2} d t^{2}+e^{(2 \sqrt{3} \gamma)} d r^{2}+e^{(-2 \sqrt{3} \gamma)} e^{(-2 \sqrt{3} \Omega)}\left(d \theta^{2}+\sin ^{2} \theta d \varphi^{2}\right),
$$

we identify

$$
e^{-2 \sqrt{3} \gamma} e^{-2 \sqrt{3} \Omega}=t^{2}, \quad e^{2 \sqrt{3} \gamma}=\frac{2 m}{t}-1, \quad N^{2}=\left(\frac{2 m}{t}-1\right)^{-1} .
$$

This metric with the identification of the $N, \gamma$ and $\Omega$ functions is also a classical solution to Einstein's equations. The WDW equation for the KS metric — with some particular factor ordering-is

$$
\left[-\frac{\partial^{2}}{\partial \Omega^{2}}+\frac{\partial^{2}}{\partial \gamma^{2}}+48 e^{-2 \sqrt{3} \Omega}\right] \psi(\Omega, \gamma)=0
$$

In [29], the authors use the WDW equation as a quantum equation for the Schwarzschild black hole, and used it as the starting point to calculate the entropy of black holes [27].

Deformed phase space black holes satisfy a deformed Poisson algebra. To construct the deformed algebra, we will follow the approach in [5]. We start with a transformation on the classical phase space variables $\left\{\Omega, \gamma, P_{\Omega}, P_{\gamma}\right\}$, which satisfies the usual Poisson algebra

$$
\begin{aligned}
\hat{\Omega} \rightarrow \Omega-\frac{\theta}{2} P_{\gamma}, & \hat{\gamma} \rightarrow \gamma+\frac{\theta}{2} P_{\Omega}, \\
\hat{P}_{\Omega} \rightarrow P_{\Omega}+\frac{\eta}{2} \gamma, & \hat{P}_{\gamma} \rightarrow P_{\gamma}-\frac{\eta}{2} \Omega .
\end{aligned}
$$

$\hat{\Omega}, \hat{\gamma}, \hat{P}_{\Omega}, \hat{P}_{\gamma}$ are the deformed phase space variables, and $\theta, \eta$ are the deformation parameters. It is easy to show that the deformed minisuperspace variables satisfy the new algebra

$$
[\hat{\Omega}, \hat{\gamma}]=i \theta, \quad\left[\hat{\Omega}, \hat{P}_{\Omega}\right]=\left[\hat{\gamma}, \hat{P}_{\gamma}\right]=i+\sigma, \quad\left[\hat{P}_{\Omega}, \hat{P}_{\gamma}\right]=i \eta,
$$

where $\sigma=\theta \eta / 4$. Now that we have introduced the deformed phase space, we use the Hamiltonian constraint for the KS model, but constructed with the variables that satisfy the modified algebra. When working with phase space deformations, two physical descriptions arise. One is based on the original variables $\Omega, \gamma$, and another is based on the new variables $\hat{\Omega}, \hat{\gamma}$. The first description has the interpretation of a commutative theory, where the effects of the deformation are encoded in a modified interaction; this description is referred as the " $C$-frame" interpretation. The second theory privileges the deformed variables; this is a theory with noncommutative variables, but with the original interaction, and is usually referred as the "NC-frame" formulation. In this paper, we will work in the "C-frame". Therefore, we can use a commutative space where the noncommutative effects are encoded in a modified potential $[30,31]$. This allows us to use the Feynman-Hibbs method to calculate the entropy of the deformed phase space black hole.

With the transformations (6), the WDW equation is deformed and exhibits an explicit dependence on the noncommutative parameters

$$
\left[-\left(-i \frac{\partial}{\partial \Omega}+\frac{\eta}{2} \gamma\right)^{2}+\left(-i \frac{\partial}{\partial \gamma}-\frac{\eta}{2} \Omega\right)^{2}-48 e^{-2 \sqrt{3}\left[\Omega+\frac{i \theta}{2} \frac{\partial}{\partial \gamma}\right]}\right] \psi(\Omega, \gamma)=0 .
$$

We use the anzats $\psi_{v}(\Omega, \gamma)=\Xi(\Omega) e^{\left[i\left(v-\frac{\eta}{2} \Omega\right)\right] \gamma}$ in Equation (8) to obtain

$$
-\Xi^{\prime \prime}(\Omega)-(\eta \Omega-v)^{2} \Xi(\Omega)+48 \exp [-2 \sqrt{3}(1+\sigma) \Omega+\sqrt{3} \theta v] \Xi(\Omega)=0 .
$$

This second-order ordinary differential equation can be solved numerically, and the solutions depend on the deformation parameters $\theta$ and $\eta$. For our purposes, we do not need analytical 
solutions. To compute the partition function of the deformed black hole, we use the Feynman-Hibbs procedure [26]. This procedure is based on exploiting the similarities of the expression of the density matrix and the kernel of Feynman's path integral approach to quantum mechanics. By doing a Wick rotation $t \rightarrow i \beta$, we get the Boltzmann factor, and the kernel is transformed to the density matrix. The kernel is calculated along the paths that go from $x_{1}$ to $x_{2}$, if we consider small $\Delta t$ (small $\beta$ ). Then when calculating the partition function, only the paths that stay near $x_{1}$ have a non-negligible contribution (the exponential in the expression for the density matrix gives a negligible contribution to the sum from the other paths). Therefore, the potential to a first-order approximation can be written as $V(x) \approx V\left(x_{1}\right)$ for all the contributing paths. In this approximation, we can formally establish a map from the path integral formulation of quantum mechanics to the classical canonical partition function. To introduce quantum mechanical effects, we must incorporate the changes to the potential along the path; in particular, we are interested in the first-order effects. For this, we start by doing a Taylor expansion around the mean position $\tilde{x}$ along any path. Calculating the kernel with $\tilde{x}$ and doing the Wick rotation, we get the modified partition function. This partition function is calculated in a classical manner, but with the corrected potential, and the quantum effects are encoded in the corrected potential (the potential calculated along the mean value of the path). The effective potential is a mean value of the potential $V(x)$ averaged over points near $\tilde{x}$ with a Gaussian distribution. Therefore, to incorporate the quantum effects, we only need to calculate the corrected potential, and simply calculate the partition function using the corrected potential $[23,27]$. Because we are using the "C-frame" interpretation for the deformed phase space model, we can assume that we are working with commutative variables, and the noncommutative effects are encoded in the potential

$$
V(\Omega)=48 e^{[-2 \sqrt{3}(1+\sigma) \Omega+\sqrt{3} \theta v]}-(\eta \Omega-v)^{2} .
$$

Furthermore, to calculate the canonical partition function, we can use the Feynman-Hibbs approach to the noncommutative potential $[23,24,27]$. We start by expanding the potential to second order in $\Omega$ and making the change of variable $\Omega \rightarrow \frac{1}{\sqrt{6}} \chi+b$, where $b$ is a constant:

$$
b=\frac{a \eta-48 \sqrt{3}(1+\sigma) e^{\sqrt{3} \theta a}}{\eta^{2}-288(1+\sigma)^{2} e^{\sqrt{3} \theta a}} .
$$

Now, multiplying by Planck's energy $E_{p}$ and setting $\chi=\frac{x}{l_{p}}$ Equation (9) takes the form

$$
\begin{aligned}
& -\frac{l_{p}^{2} E_{p}}{2} \Xi^{\prime \prime}(x)+\left(4(1+\sigma)^{2} e^{\sqrt{3} \theta v}-\frac{\eta^{2}}{72}\right) \frac{E_{p}}{l_{p}^{2}} x^{2} \Xi(x) \\
& =\frac{E_{p}}{12}\left[v^{2}+\frac{\left(v \eta-48 \sqrt{3}(1+\sigma) e^{\sqrt{3} \theta v}\right)^{2}}{288(1+\sigma)^{2} e^{\sqrt{3} \theta v}-\eta^{2}}-48 e^{\sqrt{3} \theta v}\right] \Xi(x) .
\end{aligned}
$$

The Feynman-Hibbs procedure allows the incorporation of the quantum corrections to the partition function through the corrected potential, which results in

$$
U(x)=\frac{3}{4 \pi}\left((1+\sigma)^{2} e^{\sqrt{3} \theta v}-\frac{\eta^{2}}{288}\right) \frac{E_{p}}{l_{p}^{2}}\left[x^{2}+\frac{\beta l_{p}^{2} E_{p}}{12}\right] .
$$

The corrected partition function is

$$
Z=\sqrt{\frac{2 \pi}{3}}\left((1+\sigma)^{2} e^{\sqrt{3} \theta v}-\frac{\eta^{2}}{288}\right)^{-\frac{1}{2}} \frac{e^{-\frac{1}{16 \pi}\left((1+\sigma)^{2} e^{\sqrt{3} \theta v}-\frac{\eta^{2}}{288}\right) \beta^{2} E_{p}^{2}}}{\beta E_{p}}
$$


from which we can proceed to calculate thermodynamic properties.

The internal energy is $\langle E\rangle=-\frac{\partial}{\partial \beta} \ln Z$ :

$$
\langle E\rangle=\frac{1}{\beta}+\frac{1}{8 \pi}\left((1+\sigma)^{2} e^{\sqrt{3} \theta v}-\frac{\eta^{2}}{288}\right) \beta E_{p}^{2} .
$$

For the black hole $\langle E\rangle=m c^{2}$, and solving the quadratic equation for $\beta$, we get the temperature of the deformed phase space black hole. The temperature of the black hole can be written in terms of the deformation parameters and mass of the black hole and defining the "noncommutative" temperature $\hat{\beta}_{H}$

$$
\hat{\beta}_{H}=\frac{8 \pi m c^{2}}{\left((1+\sigma)^{2} e^{\sqrt{3} \theta v}-\frac{\eta^{2}}{288}\right) E_{p}^{2}},
$$

then we can write a simple expression for the deformed phase space black hole temperature.

$$
\beta=\hat{\beta}_{H}\left(1-\frac{1}{m c^{2} \hat{\beta}_{H}}\right) .
$$

It is easy to verify that the appropriate values of the deformation parameter reproduce results in $[23,27,32]$. Usually, the temperature is defined by the elimination of conical singularities at the horizon of the Euclidean black hole geometry. In order to determine the temperature of the horizon of the deformed black hole, we need to take the full noncommutative gravity action (which is highly non-linear [14-19]), find the deformed black hole metric, and calculate the temperature by resolving the conical singularity. Due to the complexity of noncommutative theories of gravity, we choose to follow the approach of noncommutative cosmology: introduce noncommutativity in the minisuperspace. We are using a well-defined formalism to calculate the thermodynamics of the black hole that in the commutative case gives the correct temperature $[23,27,32]$. Therefore, we can be confident that the calculated temperature for the deformed phase space black hole is well-defined in the context of the Feynman-Hibbs approach, but we can not prove that it is equivalent to the removal of a conical singularity.

The entropy is calculated from $S=k \beta\langle E\rangle+k \ln Z$, and we find the deformed Hawking-Bekenstein entropy as

$$
\frac{S}{k}=\frac{\hat{S}_{B H}}{k}-\frac{1}{2} \ln \left[\frac{\hat{S}_{B H}}{k}\right]+\mathcal{O}\left[\left(\frac{\hat{S}_{B H}}{k}\right)^{-1}\right],
$$

where

$$
\frac{\hat{S}_{B H}}{k}=\frac{4 \pi m^{2} c^{4}}{\left((1+\sigma)^{2} e^{\sqrt{3} \theta v}-\frac{\eta^{2}}{288}\right) E_{p}^{2}},
$$

as with the temperature, for $\theta=0$ and $\eta=0$, we recover the commutative result. For $\eta=0$ and $\theta \neq 0$, we obtain the results in [23]. Finally, when the deformation is only present on the canonical momentum $(\theta=0$ and $\eta \neq 0)$, with $E_{p}=c=k=1$, we get

$$
S_{B H}^{\eta}=4 \pi m^{2}\left(1+\frac{\eta^{2}}{288}+\ldots\right)
$$

to first order in the $(\eta)^{2}$.

As expected, the phase space deformation gives a correction to the Schwarzschild black hole entropy. 


\section{Deformed Phase Space Parameters and $\Lambda$}

During the last few years, mounting evidence on the relationship between $\Lambda$ and the deformation parameter $\beta$ has appeared [4-6]. To find an expression for $\Lambda_{e f f}$, the authors compare the de Sitter cosmology scale factor with the scale factor of the deformed phase space model in the limit $t \rightarrow \infty$. In this limit, the scale factor of the deformed model behaves as the de Sitter scale factor, and an effective cosmological constant $\Lambda_{e f f}$ can be defined. This effective cosmological constant is a function of the deformation parameters. In order to obtain the relationship between the deformation parameters and the cosmological constant, we will compare a commutative model that includes $\Lambda$ with a deformed phase space model that does not include $\Lambda$. To establish the connection between $\Lambda$ and the parameter $\eta$, we choose the Schwarzschild-de Sitter black hole as the commutative gravitational system with a cosmological constant.

The metric for the Schwarzschild-de Sitter black hole model is

$$
d s^{2}=-\left(1-\frac{2 m}{r}+\frac{\Lambda r^{2}}{3}\right) d t^{2}+\frac{d r^{2}}{1-\frac{2 m}{r}+\frac{\Lambda r^{2}}{3}}+r^{2}\left(\mathrm{~d} \theta^{2}+\sin ^{2} \theta \mathrm{d} \phi^{2}\right),
$$

which depends on the mass and the cosmological constant. There is a singularity at $r=0$ and two horizons. The smaller one, $r_{b}$, is called the black hole horizon, and the larger one, $r_{c}$, the cosmological horizon. As explained in [33], we have two options: either use $r_{c}$ or $r_{b}$ as boundaries. When calculating thermodynamic properties, this can be physically interpreted as two horizons that are not in thermal equilibrium [34]. For our purposes, we will restrict to the black hole horizon.

The black hole horizon radius can be written as

$$
r_{b}=\frac{2}{\sqrt{\Lambda}} \cos \left(\frac{\pi+\chi}{3}\right)
$$

where $\chi=\cos ^{-1}\left(3 \sqrt{\Lambda m^{2}}\right)$.

The mass can be defined in terms of the black hole horizon [35,36]:

$$
m=\frac{r_{b}}{2}\left(1-\frac{\Lambda r^{2}}{3}\right)
$$

$m$ is only defined when $r$ takes values between the two horizons; for vanishing $\Lambda$, we get the value for Schwarzschild black hole.

To calculate the entropy, we follow as usual; thus, the entropy is proportional to the area $S \sim \pi r_{b}^{2}$. Expanding the black hole radius $r_{b}$ to third order in $\sqrt{\Lambda} m$, we get for the entropy

$$
S=\frac{\pi r_{b}^{2}}{4} \approx \pi m^{2}\left(1+\frac{8 \Lambda m^{2}}{3}\right)+\cdots
$$

As already stated, we are interested in comparing the deformed phase space Schwarzschild black hole with the commutative Schwarzschild-de Sitter black hole. Comparing (23) with the entropy of the deformed model (19), we find that $\Lambda \sim \frac{\eta^{2}}{m^{2}}$.

\section{Conclusions}

In this paper, we have explored the possible relationship between the cosmological constant $\Lambda$ and the noncommutative deformation parameter of the canonical momentum. This relationship has been suggested when studying the late time behaviour of several cosmological models and comparing with the commutative de Sitter cosmology [5]. The results point out a quadratic dependence of the cosmological constant with the noncommutative parameter, suggesting that the origin of the cosmological constant could be related to phase space deformations. 
The approach we followed is similar; we compare the deformed phase space Schwarzschild black hole with the commutative Schwarzschild-de Sitter black hole. We take advantage of the relationship between the Schwarzschild metric and the KS cosmological model so that we can use the temperature and entropy of the deformed phase space black hole, calculated from the deformed KS-WDW equation [24]. Comparing with the entropy of the Schwarzschild-de Sitter black hole in the limit $\eta<<1$ and $\theta=0$, we find that $\Lambda \sim \frac{\eta^{2}}{m^{2}}$. This is consistent with the results obtained in the cosmological scenario [5], where a quadratic relationship between the cosmological constant and the deformation parameter has been obtained. It is encouraging that we have obtained the same result in a different gravitational system, but we cannot say that the deformation parameter can always replace $\Lambda$. We need to establish the relationship between $\Lambda$ and $\eta$ under less-restrictive conditions. This is under research, and will be reported elsewhere.

Acknowledgments: This work is supported by CONACyT grants 167335, 179208, 257919, 290649 and DAIP1107/2016 and by Centro Universitario de la Ciénega de la U. de G. project Desarrollo de la investigación y fortalecimiento del posgrado 235506, Andrés Crespo-Hernández is supported by CONACyT PhD. grant.

Author Contributions: All of the authors conceived, designed and performed the calculations together. In addition, they analyzed the results and wrote the paper together. All authors have read and approved the final manuscript. The authors contributed to this work equally.

Conflicts of Interest: The authors declare no conflict of interest.

\section{References}

1. Polchinski, J. The cosmological constant and the string theory landscape. arXiv 2006, arXiv:hep-th/0603024.

2. Burgess, C.P. The Cosmological Constant Problem: Why it's hard to get Dark Energy from Micro-physics. arXiv 2013, arXiv:1309.4133.

3. Obregon, O.; Quiros, I. Can noncommutative effects account for the present speed up of the cosmic expansion? Phys. Rev. 2011, 84, 044005.

4. Vakili, B.; Pedram, P.; Jalalzadeh, S. Late time acceleration in a deformed phase space model of dilaton cosmology. Phys. Lett. 2010, 687, 119-123.

5. Pérez-Payan, S.; Sabido, M.; Yee-Romero, C. Effects of deformed phase space on scalar field cosmology. Phys. Rev. D 2013, 88, 027503.

6. Malekolkalami, B.; Atazadeh, K.; Vakili, B. Late time acceleration in a non-commutative model of modified cosmology. Phys. Lett. B 2014, 739, 400-404.

7. Obregon, O.; Sabido, M.; Mena, M. On noncommutative minisuperspace, cosmology and Lambda. Mod. Phys. Lett. A 2009, 24, 1907-1914.

8. Wigner, E. On the Quantum Correction for Thermodynamic Equilibrium. Phys. Rev. 1932, 40, 749.

9. Connes, A. Noncommutative geometry Year 2000. arXiv 2000, arXiv:math/0011193.

10. Connes, A. A Short survey of noncommutative geometry. J. Math. Phys. 2000, 41, 3832-3866.

11. Seiberg, N.; Witten, N. String theory and noncommutative geometry. J. High Energy Phys. 1999, 9909, 032.

12. Connes, A.; Douglas, M.R.; Schwarz, A.S. Noncommutative geometry and matrix theory: Compactification on tori. J. High Energy Phys. 1998, 9802, 003.

13. Douglas, M.R.; Nekrasov, N.A. Noncommutative field theory. Rev. Mod. Phys. 2001, 73, 977.

14. Garcia-Compean, H.; Obregon, O.; Ramirez, C.; Sabido, M. Noncommutative selfdual gravity. Phys. Rev. D 2003, 68, 044015.

15. Garcia-Compean, H.; Obregon, O.; Ramirez, C.; Sabido, M. Noncommutative topological theories of gravity. Phys. Rev. D 2003, 68, 045010.

16. Aschieri, P.; Dimitrijevic, M.; Meyer, F.; Wess, J. Noncommutative geometry and gravity. Class. Quant. Grav. 2006, 23, 1883.

17. Calmet, X.; Kobakhidze, A. Noncommutative general relativity. Phys. Rev. D 2005, 72, 045010.

18. Alvarez-Gaume, L.; Meyer, L.; Vazquez-Mozo, M.A. Comments on noncommutative gravity. Nucl. Phys. B 2006, 753, 92-117.

19. Estrada-Jimenez, S.; Garcia-Compean, H.; Obregon, O.; Ramirez, C. Twisted Covariant Noncommutative Self-dual Gravity. Phys. Rev. D 2008, 78, 124008. 
20. Chamseddine, A.H.; Connes, A. Noncommutative Geometry as a Framework for Unification of all Fundamental Interactions including Gravity. Part I. Fortschr. Phys. 2010, 58, 553-600.

21. Garcia-Compean, H.; Obregon, H.; Ramirez, H. Noncommutative quantum cosmology. Phys. Rev. Lett. 2002, $88,161301$.

22. Rezaei-Aghdam, A.; Darabi, F.; Rastkar, A.R. Noncommutativity in quantum cosmology and the cosmological constant problem. Phys. Lett. 2005, 615, 141-145.

23. Lopez-Dominguez, J.C.; Obregon, O.; Sabido, M.; Ramirez, C. Towards Noncommutative Quantum Black Holes. Phys. Rev. D 2006, 74, 084024.

24. Bastos, C.; Bertolami, O.; Dias, N.C.; Prata, J.N. Black Holes and Phase Space Noncommutativity. Phys. Rev. D 2009, 80, 124038.

25. Bastos, C.; Bertolami, O.; Dias, N.C.; Prata, J.N. Phase-Space Noncommutative Quantum Cosmology. Phys. Rev. D 2008, 78, 023516.

26. Feynman, R.P.; Hibbs, A.R. Quantum Mechanics and Path Integrals; McGraw-Hill: New York, NY, USA, 1965.

27. Obregon, O.; Sabido, M.; Tkach, V.I. Entropy using path integrals for quantum black hole models. Gen. Relativ. Grav. 2001, 33, 913-919.

28. Kantowski, R.; Sachs, R.K. Some Spatially Homogeneous Anisotropic Relativistic Cosmological Models. J. Math. Phys. 1966, 7, 443-446.

29. Obregón, O.; Ryan, M.P. Quantum Planck Size Black Hole States Without a Horizon. Mod. Phys. Lett. A 1998, 13, 3251-3258.

30. Barbosa, G.D. Noncommutative conformally coupled scalar field cosmology and its commutative counterpart. Phys. Rev. D 2005, 71, 063511.

31. Perez-Payan, S.; Sabido, M.; Mena, E.; Yee-Romero, C. Analysis of Scalar Field Cosmology with Phase Space Deformations. Adv. High Energy Phys. 2014, 2014, 958137.

32. Kastrup, H.A. Canonical quantum statistics of an isolated Schwarzschild black hole with a spectrum $E_{n}=\sigma n E_{p}$. Phys. Lett. B 1997, 413, 267-281.

33. Teitelboim, C. Gravitational thermodynamics of Schwarzschild-de Sitter space. arXiv 2002, arXiv:hep-th/0203258.

34. Gomberoff, A.; Teitelboim, C. De Sitter black holes with either of the two horizons as a boundary. Phys. Rev. D 2003, 67, 104024 .

35. Bousso, R. Bekenstein bounds in de Sitter and flat space. J. High Energy Phys. 2001, 0104, 035.

36. Lopez-Dominguez, J.C.; Obregon, O.; Zacarias, S. Towards a supersymmetric generalization of the Schwarzschild-(anti) de Sitter space-times. Phys. Rev. D 2011, 84, 024015.

(c) 2017 by the authors. Licensee MDPI, Basel, Switzerland. This article is an open access article distributed under the terms and conditions of the Creative Commons Attribution (CC BY) license (http:/ / creativecommons.org/licenses/by/4.0/). 